\title{
Quasi-2D Optomechanical Crystal Cavity for Quantum Optomechanics
}

\author{
Hengjiang Ren, ${ }^{1}$ Gregory S. MacCabe, ${ }^{1}$ Jie Luo, ${ }^{1}$ Hannes Pfeifer, ${ }^{2}$ \\ Andrew J. Keller, ${ }^{1}$ and Oskar Painter ${ }^{1, *}$ \\ ${ }^{1}$ Institute for Quantum Information and Matter and Thomas J. Watson, Sr., Laboratory of Applied Physics, \\ California Institute of Technology, Pasadena, USA \\ ${ }^{2}$ Max Planck Institute for the Science of Light, Staudtstrasse 2, 91058 Erlangen, Germany
}

opainter@caltech.edu

\begin{abstract}
We present the design and characterization of a quasi-two-dimensional optomechanical crystal cavity. At a refrigerated temperature of $10 \mathrm{mK}$, an intrinsic mechanical quality factor of 1.2 billion is observed and an effective quantum cooperativity greater than unity is realized under steady-state optical pumping. (C) 2019 The Author(s)

OCIS codes: $220.4880,230.5298,270.5585$
\end{abstract}

Optomechanical crystals (OMCs), corresponding to a combination of photonic and phononic crystal, can be fabricated into the thin-film surface of a silicon microchip and engineered to yield strong optomechanical interactions due to the co-localization of optical and mechanical fields. Previous work has realized one-dimensional silicon nanobeams with $\sim 1 \mathrm{MHz}$ vacuum optomechanical coupling rate, enabling a number of quantum optomechanics experiments, from laser cooling of mechanical motion to the quantum ground state [1] to optomechanical Bell tests [2]. A significant roadblock to further application of silicon nanobeams for quantum applications is the very weak, yet non-negligible parasitic optical absorption in current devices [2,3]. Optical absorption at sub-bandgap $(\sim 1500 \mathrm{~nm})$ wavelengths in silicon nanobeam devices, thought to occur due to surface defect states, can yield significant heating of the microwave $(\sim 3-6 \mathrm{GHz})$ acoustic mode of the device. At cryogenic temperatures $(\lesssim 1 \mathrm{~K})$ this leads to an elevated phonon bath occupancy of $n_{\mathrm{b}} \approx 10$ on a microsecond timescale upon applying an optical pulse power at a level corresponding to a single intra-cavity photon $\left(n_{\mathrm{c}}\right)$ [3]. Moreover, this hot bath persists even after the removal of the light field for times on order several microseconds to milliseconds. The 'quantum' cooperativity $\left(C_{\mathrm{eff}}\right)$, corresponding to the standard photon-phonon cooperativity divided by the Bose factor of the thermal
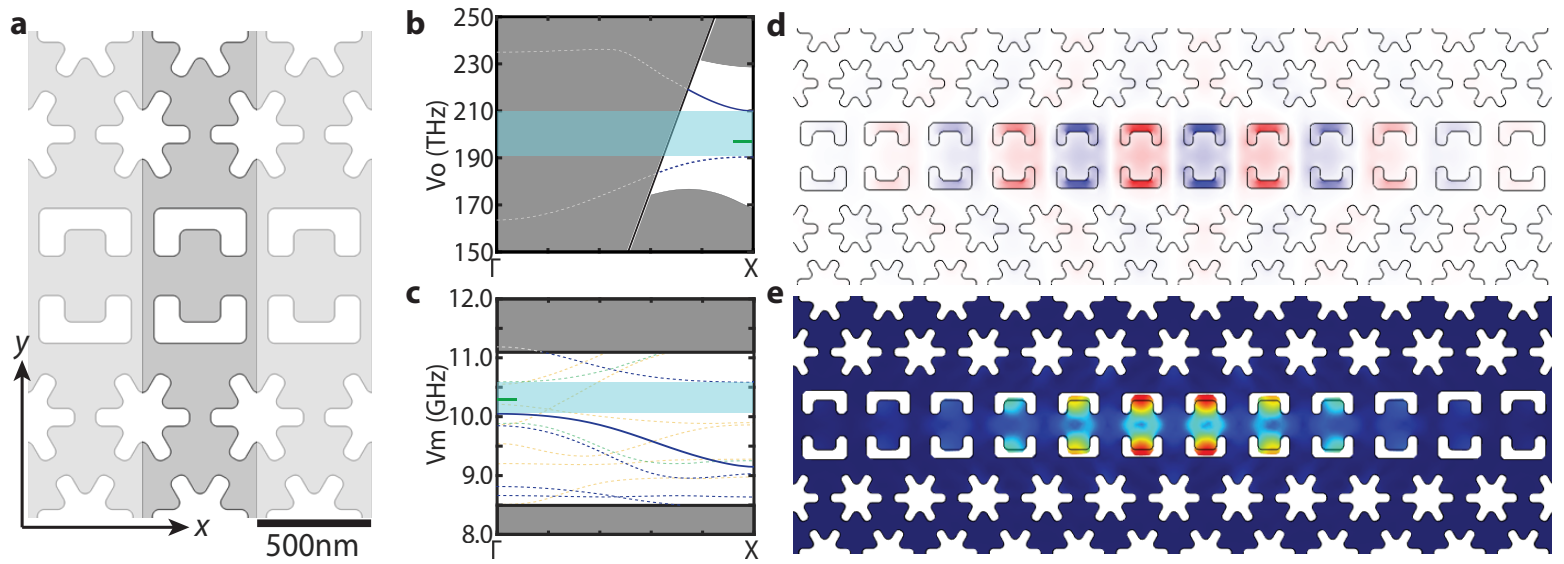

Fig. 1. a, Unit cell schematic of a linear waveguide formed in the snowflake crystal. Guided modes of the waveguide propagate along the $x$-axis. $\mathbf{b}$, Photonic and $\mathbf{c}$, phononic band structure of the linear waveguide (only modes of even symmetry about the center of the silicon slab are shown for photonic band structure). The solid blue curves are the waveguide bands of interest; green and yellow dashed lines are the guided modes of other symmetries; shaded light blue regions are bandgaps of interest; green tick mark indicates the cavity mode frequencies; gray regions denote the continua of propagating modes outside of the snowflake crystal bandgap. d, FEM-simulated mode profile ( $E_{y}$ component of the electric field) of the fundamental optical resonance $\omega_{o} / 2 \pi=194 \mathrm{THz}$. e, FEM-simulated displacement profile of fundamental acoustic resonance at $\omega_{m} / 2 \pi=10.27 \mathrm{GHz}$. 

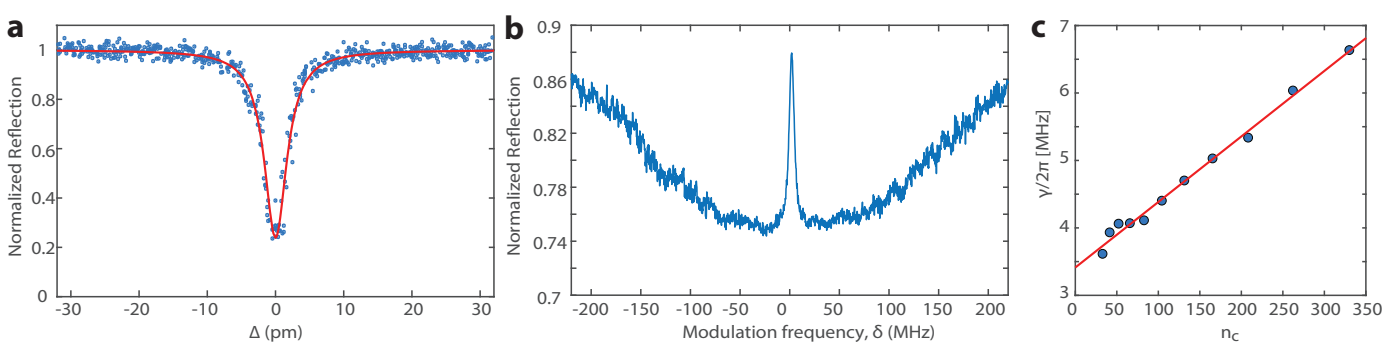

Fig. 2. Characterization of a quasi-2D OMC device at room temperature. a, Normalized wavelength scan of the optical mode of a quasi-2D OMC device. b. Normalized EIT scan of the mechanical mode of interest centered around $\omega_{m, 0} / 2 \pi=10.21 \mathrm{GHz}\left(\delta \equiv \omega-\omega_{m, 0}\right)$. c, Mechanical mode linewidth versus the number of intracavity photons, $n_{\mathrm{c}}$.

bath, is thus limited to values lower than unity for all but a microsecond or so around the time an optical pulse is applied. Two-dimensional (2D) OMCs, with their significantly increased thermal connection to the surrounding environment compared to $1 \mathrm{D}$ nanobeams, offer a promising route to realizing $C_{\text {eff }}>1$ in the steady-state. Initial work in this direction utilized snowflake-shaped holes in a silicon membrane to create a quasi-2D OMC with substantially higher optical power handling capabilities than nanobeams [4]. However, in the original snowflake design a relatively low optomechanical coupling of $g_{0} / 2 \pi=220 \mathrm{kHz}$ was obtained.

Here we demonstrate an improved silicon $2 \mathrm{D}$ OMC with a factor $\times 53$ improvement in back-action per photon. Our new OMC cavity design is created from a snowflake crystal with both acoustic and optical bandgaps by replacing one row of snowflakes with repeated ' $C$ ' shapes. The ' $C$ ' shape better focuses the optical mode at the air-silicon boundary, greatly increasing the moving boundary contribution to the optomechanical coupling. The line defect in the snowflake lattice acts as a waveguide for photons and phonons within their bandgap, as shown in Fig. 1b-1c. The properties of the waveguide are then modulated along its length, locally shifting the bands to frequencies that cannot propagate within the waveguide to create localized resonances. Simulated field profiles of the fundamental optical resonance $\left(\omega_{o} / 2 \pi=194 \mathrm{THz}, \lambda_{o}=1550 \mathrm{~nm}\right)$ and the strongly coupled mechanical resonance $\left(\omega_{m} / 2 \pi=10.27 \mathrm{GHz}\right)$ of such a $2 \mathrm{D}$ OMC are shown in Fig. 1d and 1e. The localized mechanical mode has a theoretical vacuum optomechanical coupling rate of $g_{0} / 2 \pi=1.4 \mathrm{MHz}$ to the co-localized optical resonance.

We fabricated devices based upon this design and measured them at both room temperature and in a dilution refrigerator environment. From room temperature measurements (see Fig. 2) we determine the wavelength of the fundamental optical resonance of the 2D OMC to be $\lambda_{o}=1558.8 \mathrm{~nm}$, with a loaded (intrinsic) optical $Q$-factor of $Q_{t}=3.90 \times 10^{5}\left(Q_{i}=5.30 \times 10^{5}\right)$. We measure the optomechanically coupled acoustic resonance using a pumpprobe scheme with optical pump frequency at the red motional sideband of the optical cavity, from which we find a mechanical frequency of $\omega_{m} / 2 \pi=10.21 \mathrm{GHz}$. From fitting the mechanical damping rate, $\gamma$, versus applied intra-cavity photon $n_{\mathrm{c}}$, we extract a vacuum coupling rate of $g_{0} / 2 \pi=1.09 \mathrm{MHz}$.

Heating, backaction cooling, and damping dynamics of quasi-2D OMCs are also measured in a dilution refrigerator at a base temperature of $T_{f} \approx 10 \mathrm{mK}$. Steady-state back-action cooling below the single phonon level is observed indicating that $C_{\text {eff }}>1$. Through phononic bandgap shielding an intrinsic mechanical damping rate of $\gamma_{i} / 2 \pi=8.2 \mathrm{~Hz}\left(Q_{m} \approx 1.2 \times 10^{9}\right)$ is also realized. Up-to-date dilution refrigerator measurements, including optical-absorption-induced bath dynamics and quantum cooperativity versus optical power, will be presented.

\section{References}

1. J. Chan, T. P. M. Alegre, A. H. Safavi-Naeini, J. T. Hill, A. Krause, S. Gröblacher, M. Aspelmeyer, and O. Painter, "Laser cooling of a nanomechanical oscillator into its quantum ground state," Nature 478, 8992 (2011).

2. I. Marinković, A. Wallucks, R. Riedinger, S. Hong, M. Aspelmeyer, and S. Gröblacher, "Optomechanical bell test," Phys. Rev. Lett. 121, 220404 (2018).

3. S. M. Meenehan, J. D. Cohen, G. S. MacCabe, F. Marsili, M. D. Shaw, and O. Painter, "Pulsed Excitation Dynamics of an Optomechanical Crystal Resonator near Its Quantum Ground State of Motion," Physical Review X 5, 041002 (2015).

4. A. H. Safavi-Naeini, J. T. Hill, S. Meenehan, J. Chan, S. Gröblacher, and O. Painter, "Two-dimensional phononic-photonic bandgap optomechanical crystal cavity," Phys. Rev. Lett. 112, 153603 (2014). 